\title{
Effects of dimethylolpropionic acid modification on the characteristics of polyethylene terephthalate fibers
}

\author{
ZHAOSONG HUANG $^{1 *}$, LONG BI ${ }^{2 *}$, ZHENYU ZHANG $^{2}$ and YISHENG HAN ${ }^{2}$ \\ ${ }^{1}$ Institute of Orthopaedics, The 323rd Hospital of PLA; ${ }^{2}$ Institute of Orthopaedics, Xijing Hospital, \\ The Fourth Military Medical University, Xi'an, P.R. China
}

Received December 19, 2011; Accepted March 19, 2012

DOI: $10.3892 / \mathrm{mmr} .2012 .1012$

\begin{abstract}
Polyethylene terephthalate (PET) fibers are widely used in the preparation of artificial ligaments. However, due to their lack of hydrophilicity, PET fibers have low biocompatibility, which usually results in the poor biological activity of the products. In the present study, in order to improve the hydrophilicity and biocompatibility of PET fibers, we modified their surface using dimethylolpropionic acid (DMPA). Scanning electron microscopy (SEM), Fourier transform infrared spectroscopy (FTIR), differential scanning calorimetry (DSC), X-ray diffraction (XRD), tensile testing and cell culture were employed to observe the effects of DMPA modification on the characteristics of the DMPA-PET fibers. In contrast to the original PET fibers, the surface of the DMPA-PET fibers became rough as demonstrated by SEM. The FTIR spectrum further confirmed that a number of hydrophilic groups were formed on the surface of DMPA-PET. However, there were no significant changes in crystallinity and tensile strength between the PET and the DMPA-PET fibers as revealed by DSC and XRD (P>0.05). Finally, the cell co-culture test revealed that the adhesion and proliferation of bone marrow-derived stromal cells increased greatly on the DMPA-PET fibers compared to those on the original PET fibers $(\mathrm{P}<0.05)$. These results demonstrate that DMPA-PET fibers have significant potential as a material for the development of artificial ligaments.
\end{abstract}

Correspondence to: Dr Yisheng Han, Institute of Orthopaedics, Xijing Hospital, The Fourth Military Medical University, 15 West Changle Road, Xi'an 710032, P.R. China

E-mail: 19069697@qq.com

${ }^{*}$ Contributed equally

Key words: polymers, dimethylolpropionic acid, hydrophilicity, strength

\section{Introduction}

Anterior cruciate ligament (ACL) ruptures are frequently encountered in clinical practice, and can lead to the dysfunction of injured joints, increased risk of subsequent injury of the meniscus and cartilage and even increased risk of early osteoarthritis (1-4). Due to the lack of blood supply, ACL ruptures cannot heal independently, and therefore surgical reconstruction or replacement is necessary $(5,6)$. Autologous grafts, including quadricep, patellar and hamstring tendon grafts and iliotibial bands or tracts, are commonly used in intra-articular replacements of ACL (2,7-9). However, certain well-recognized drawbacks, including delayed recovery of the intra-articular donor site, pain in the anterior part of the knee, reduced strength of the tendon at the harvesting site, and lengthy surgery required for harvesting the grafts, restrict the application of autografts $(10,11)$. Allografts are usually used in lower-demand patients or patients who are undergoing revision ACL surgery if an ACL reconstruction fails (12). However, these grafts are of poor strength and carry a significant risk of disease transmission $(13,14)$.

In order to overcome such drawbacks, artificial ligaments have been widely studied over the past 50 years and have become a potential choice for reconstructing torn ACLs (15-17). Various materials, including carbon fibers, nylon, polytetrafluoroethylene (PTFE) and polyethylene terephthalate (PET), have been employed and have contributed greatly to the diffusion of artificial ligaments $(15,18-20)$. Among these materials, PET fibers are considered to be especially adapted to fabricate artificial ligaments, due to their plasticity, flexibility and high mechanical resistance to rupturing (21). However, due to the lack of hydrophilicity, artificial ligaments made of PET fibers appear to have lower biocompatibility, and therefore cannot integrate into the surrounding host bone tissue, which can eventually lead to rupturing of the artificial ligaments and reconstruction failure in the long-term $(21,22)$.

In this study, in order to improve the biocompatibility of PET fibers, we modified them by using a hydrophilic molecule, dimethylolpropionic acid (DMPA), which contains 2 types of functional groups, 2 primary hydroxyl and 1 tertiary carboxyl group, which make polyesters particularly water soluble. We postulated that the introduction of DMPA would increase the biocompatibility of the PET fibers without comprising their other characteristics, particularly the high mechanical 
strength. In order to test this hypothesis, scanning electron microscopy (SEM), Fourier transform infrared spectroscopy (FTIR), differential scanning calorimetry (DSC), X-ray diffraction (XRD), mechanical testing and cell culture were applied to investigate these fibers.

\section{Materials and methods}

Modification of PET by DMPA. Briefly, the original PET fibers (Taiji Co., Ltd., Wuxi, China), $5 \mathrm{~cm}$ long x $22 \mu \mathrm{m}$ in diameter, were immersed in a solution of $2.5 \mathrm{~mol} / 1$ sodium hydroxide (Sigma-Aldrich, Shanghai, China) at a ratio of 1:10 $(\mathrm{w} / \mathrm{w})$ for $3 \mathrm{~h}$ at $50^{\circ} \mathrm{C}$. The PET fibers were then placed into a $75 \%(w / v)$ tolylene-2,4-diisocyanate (TDI; Sigma-Aldrich)/ acetone solution, which contained $0.25 \%$ (v/v) dibutyltin dilaurate (DBTDL; Sigma-Aldrich), at a ratio of 1:10 (w/w) for $3 \mathrm{~h}$ at $50^{\circ} \mathrm{C}$. The PET fibers were then transferred into a $36.5 \%(\mathrm{w} / \mathrm{v})$ DMPA/acetone solution (Sigma-Aldrich), which contained $0.07 \%$ (v/v) fluorite boron (Zibo Shuanglian Petroleum and Chemical Co., Ltd., Shandong, China) as an activator for $2 \mathrm{~h}$ at $20^{\circ} \mathrm{C}$. Finally, the DMPA-modified PET (DMPA-PET) fibers were washed with distilled water, air-dried and sterilized by $\gamma$ radiation at $15 \mathrm{kGy}$ for $6 \mathrm{~h}$. The original PET fibers were also sterilized and used as the control.

SEM observation. A total of 10 samples from the DMPA-PET and the PET groups were fixed on stubs with carbon tape and sputter-coated with gold in an ion coater. The surface characteristics of the samples were studied under a vacuum using SEM (S-3400N, Hitachi, Osaka, Japan) to analyze changes in the surface morphology.

FTIR evaluation. A Fourier transform infrared spectrometer (IR-200 Thermo-Nicolet 2.2, Thermo-Nicolet corporation, Madison, WI, USA) was used to detect the changes in the functional groups of the DMPA-PET and PET fibers as previously described (23). Briefly, following appropriate background subtraction, $2 \mathrm{mg}$ of freeze-dried samples of each group were mixed with dried potassium bromide, and the samples were scanned from 4,000 to $400 \mathrm{~cm}^{-1}$ by the spectrometer. Each spectrum reported is the average of at least three spectra measured in different areas of the samples $(n=10)$.

DSC evaluation. The thermal properties of DMPA-PET and PET were analyzed by a differential scanning calorimeter (DSC-2041F; Netzsch, Selb, Germany), which was equipped with an intra-cooler and a refrigerated cooling system. Indium standard was used to calibrate the DSC temperature and enthalpy scale. Nitrogen was used to purge gas through the DSC cell at the flow rate of $50 \mathrm{ml} / \mathrm{min}$ through the cooling unit. A total of $5 \mu \mathrm{g}$ of samples from the 2 groups were hermetically sealed in an aluminium pan at a heating rate of $20 \mathrm{~K} /$ $\min (\mathrm{n}=10)$ (24). Each examination was replicated three times.

$X R D$ evaluation. An X-ray diffractometer (XRD-6000, Shimazu, Japan) was used to determine the crystalline phases of DMPA-PET and PET. The generator working parameters were set at $40 \mathrm{kV}$ and $30 \mathrm{~mA}$ to create copper $\mathrm{CuK} \alpha$ radiation $(\lambda=1.542 \AA)$. The scanning scope of $2 \theta$ was $100-60^{\circ}$ and the scanning rate was $2^{\circ}$ per min. To obtain the maximum diffrac- tion intensity, the DMPA-PET and PET fibers were prepared in a number of layers to obtain thick samples (total thickness, $2 \mathrm{~mm}$ ). This measurement was performed on at least ten samples (24).

Mechanical testing. The tensile testing was performed by a materials testing machine (Instron 5565; Instron, Norwood, MA, USA) with a performance temperature of $20 \pm 2^{\circ} \mathrm{C}$, a relative humidity of $65 \pm 5 \%$ and a humidity controlling time of $4 \mathrm{~h}$. The samples were fixed with a nominal gauge length of $20 \mathrm{~mm}$ and a static pre-tension of $0.15 \pm 0.03 \mathrm{cN}$. The tensile testing was performed at a speed of $20 \mathrm{~mm} / \mathrm{min}$ until the testing fibers failed $(n=10)$. The ultimate tensile strength, tensile stress, elongation percentage at breakage and initial modulus were determined as previously described (25).

Cytocompatibility evaluation. To determine the cytocompatibility of the 2 PET fibers, bone marrow stromal cells (BMSCs) transfected with green fluorescent protein (GFP) (Provided by Dr Lu from the Cell Laboratory of the Affiliated Dental Hospital at the Fourth Military Medical University, Xi'an, China) were seeded onto the 2 fibers at a concentration of $2 \times 10^{-6} \mathrm{cell} / \mathrm{cm}^{2}$ in Dulbecco's modified Eagle's medium (DMEM, Sigma, St. Louis, MO, USA) containing $10 \%$ fetal bovine serum (FBS; HyClone, Logan, UT, USA) at $37^{\circ} \mathrm{C}$. The adhesion and growth of the BMSCs on the fibers were observed under an inverted phase-contrast fluorescence microscope after 3 and 7 days. A PicoGreen double-stranded DNA (dsDNA) Quantification kit (Molecular Probes, Eugene, OR, USA) was used to determine the proliferation of the cells cultured in the DMEM (10\% FBS) with $20 \%$ leaching liquor of each type of fiber from day 1 to 7. The total dsDNA was extracted through enzymatic digestion and assayed following the manufacturer's instructions. The proliferation of the cells in the scaffold was interpreted by changes in the quantity of the dsDNA. The leaching liquors of the fibers were prepared as previously described (26). Cells cultured in DMEM (10\% FBS) without any leaching liquor were used as the blank control $(n=10)$.

Statistical analysis. Statistical analyses were performed using SPSS software version 14.0 (SPSS Inc., Chicago, IL, USA). The data are presented as the means $\pm \mathrm{SD}$, and were compared using the non-parametric Mann-Whitney U test or the Student's t-test. $\mathrm{P}<0.05$ was considered to indicate a statistically significant difference.

\section{Results}

SEM observation. The SEM images demonstrate that the surface of the original PET fibers was smooth without any deposits (Fig. 1A). By contrast, following modification the surface of the DMPA-PET fibers became uneven, and a number of granules, 300 to $500 \mathrm{~nm}$ in diameter, were found to be deposited on the surface (Fig. 1B).

FTIR evaluation. The different infrared spectra of the PET and DMPA-PET fibers were demonstrated by FTIR evaluation. The characteristic peaks of PET appeared at 3,431 and 2,970, $1,732,1,246,1,083$ and $716 \mathrm{~cm}^{-1}$, corresponding to the hydroxyl $(-\mathrm{OH})$, methyl $\left(-\mathrm{CH}_{3}\right)$, carbonyl $(\mathrm{C}=\mathrm{O})$, and methanediyl $\left.\left(-\mathrm{CH}_{2}\right)^{-}\right)$groups, and ethers $(\mathrm{C}-\mathrm{O}-\mathrm{C})$, respectively. By contrast, 


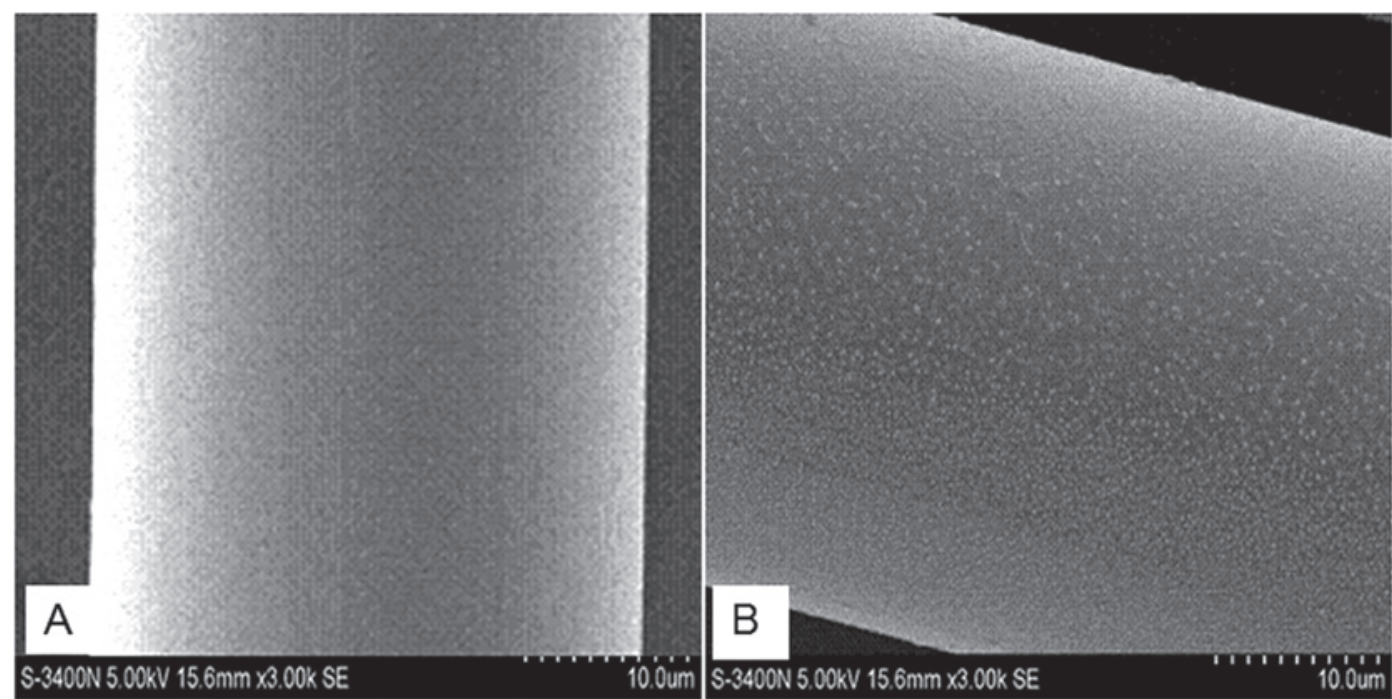

Figure 1. SEM images of the fibers. (A) PET and (B) DMPA-PET. SEM, scanning electron microscope; PET, polyethylene terephthalate; DMPA-PET, dimethylolpropionic acid-polyethylene terephthalate.
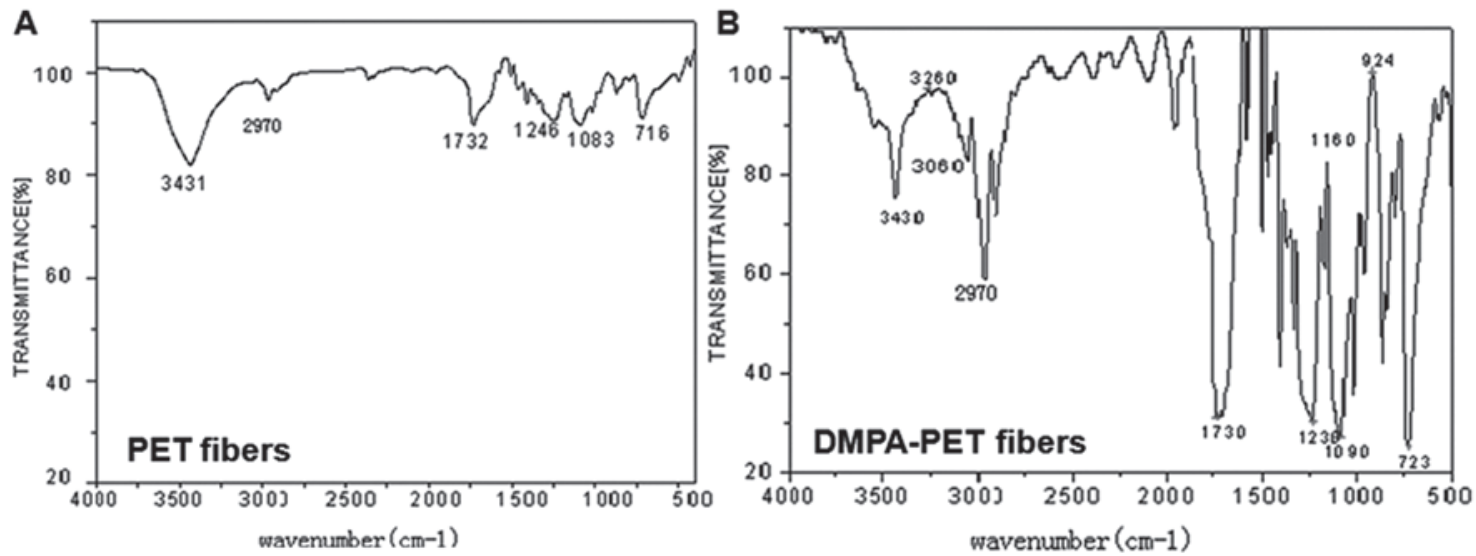

Figure 2. Infrared spectra of (A) PET and (B) DMPA-PET fibers. PET, polyethylene terephthalate; DMPA-PET, dimethylolpropionic acid-polyethylene terephthalate.

a number of newly-formed absorption bands were found in the spectrum of DMPA-PET at 3,400-3,500, 2,852-3,000 and $1,700-1,730 \mathrm{~cm}^{-1}$, which represented the newly-formed peptide bonds $(\mathrm{N}-\mathrm{H}),-\mathrm{OH}$ and carboxy $(-\mathrm{COOH})$ groups, respectively. Furthermore, the greater absorption band at the $3,200 \mathrm{~cm}^{-1}$ area in the DMPA-PET group also confirmed the increase in the hydrophilic $-\mathrm{OH}$ and $-\mathrm{COOH}$ groups (Fig. 2B).

DSC evaluation. DSC was used to characterize the thermophysical properties of the fibers. In the PET group, the first sharp endothermic peak was formed at $259.8 \pm 1.1^{\circ} \mathrm{C}$, with a fusion enthalpy of $39.5 \pm 0.5 \mathrm{~J} / \mathrm{g}$ and a crystallinity of $28.2 \pm 1.5 \%$. The second peak formed at $254.1 \pm 1.2^{\circ} \mathrm{C}$, with a fusion enthalpy of $28.0 \pm 0.5 \mathrm{~J} / \mathrm{g}$ and a crystallinity of $20.0 \pm 1.7 \%$. Similarly, the first endothermic peak of the DMPA-PET group was demonstrated at $258.1 \pm 1.2^{\circ} \mathrm{C}$, with a fusion enthalpy of $40.8 \pm 0.7 \mathrm{~J} / \mathrm{g}$ and a crystallinity of $29.2 \pm 1.6 \%$. The second peak of DMPA-PET was formed at $253.0 \pm 1.4^{\circ} \mathrm{C}$, with a fusion enthalpy of $28.8 \pm 0.6 \mathrm{~J} / \mathrm{g}$ and a crystallinity of $20.6 \pm 1.9 \%$. There were no significant differences in the endothermic peaks, fusion enthalpies and crystallinity indices between the 2 groups $(\mathrm{P}>0.05, \mathrm{n}=10$; Table I).

XRD evaluation. The XRD spectra recorded for the PET and the DMPA-PET groups are shown in Fig. 3. The XRD pattern of the PET fibers demonstrated that the original PET was mostly amorphous in nature with less crystallinity. The tip of the amorphous halo of the original PET was observed at $2 \theta=27.42 \pm 0.02^{\circ}$ in the diffraction pattern as demonstrated in the diffractogram (Fig. 3A). Only a small change was observed in the the XRD pattern of the DMPA-PET samples compared with the original one. The amorphous peak tip of the DMPA-PET samples was found to be shifted slightly towards a lower angle $\left(26.23 \pm 0.01^{\circ}\right)$, but the change was not significant $(\mathrm{n}=10, \mathrm{P}<0.05$; Fig. 3B).

Mechanical testing. As shown in Fig. 4, the ultimate tensile strength and tensile stress of the samples from the DMPA-PET group were $329.2 \pm 20.62 \mathrm{cN}$ and $0.05934 \pm 0.0038 \mathrm{MPa}$, respectively, which were similar to those from the PET group 
Table I. Thermophysical properties of the PET and DMPA-PET fibers $(n=10)$.

\begin{tabular}{llllllll}
\hline & \multicolumn{2}{c}{ Endothermic peak $\left({ }^{\circ} \mathrm{C}\right)$} & & \multicolumn{2}{c}{ Fusion enthalpy $(\mathrm{J} / \mathrm{g})$} & & \multicolumn{2}{c}{ Crystallinity $(\%)$} \\
\cline { 2 - 3 } Groups & 1st heating & 2nd heating & & 1st heating & 2nd heating & 1st heating & 2nd heating \\
\hline PET & $259.8 \pm 1.1$ & $254.1 \pm 1.2$ & & $39.5 \pm 0.5$ & $28.0 \pm 0.5$ & $28.2 \pm 1.5$ & $20.0 \pm 1.7$ \\
DMPA-PET & $258.1 \pm 1.2$ & $253.0 \pm 1.4$ & & $40.8 \pm 0.7$ & $28.8 \pm 0.6$ & $29.2 \pm 1.6$ & $20.6 \pm 1.9$ \\
\hline
\end{tabular}

PET, polyethylene terephthalate; DMPA-PET, dimethylolpropionic acid-polyethylene terephthalate.
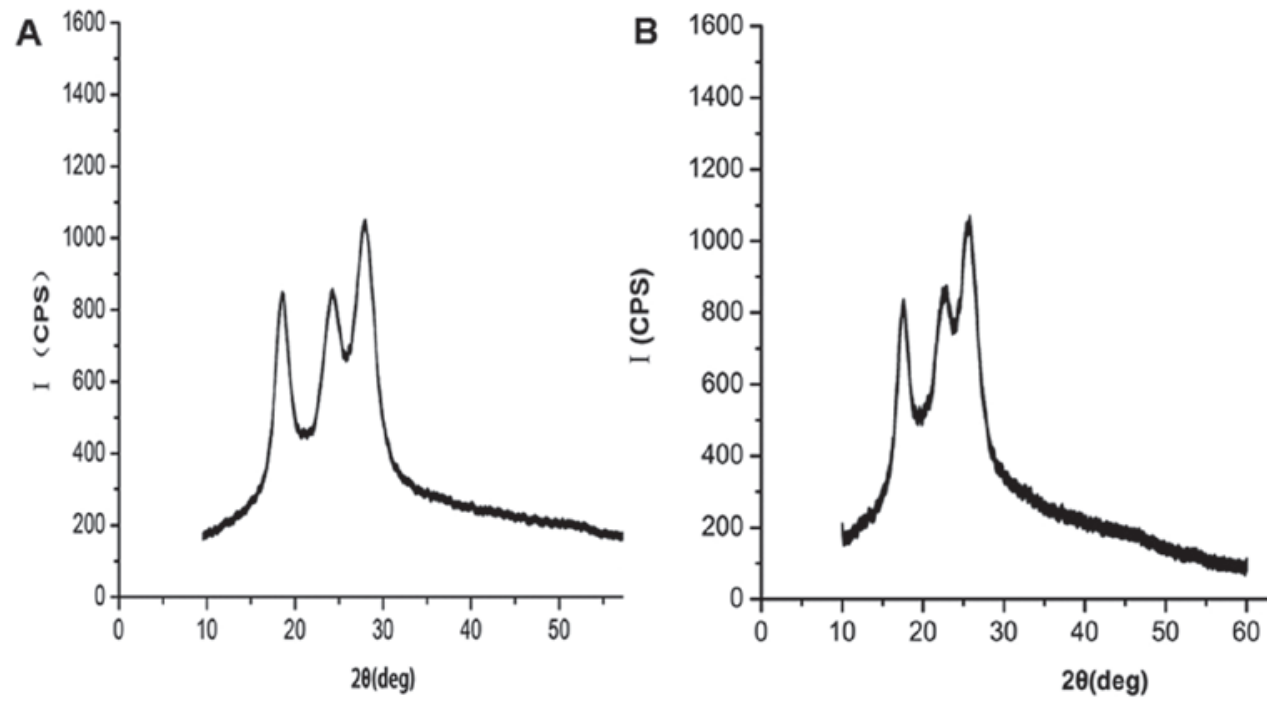

Figure 3. XRD images of the (A) PET fibers and (B) DMPA-PET fibers. CPS, counts per second; XRD, X-ray diffraction; PET, polyethylene terephthalate; DMPA-PET, dimethylolpropionic acid-polyethylene terephthalate.
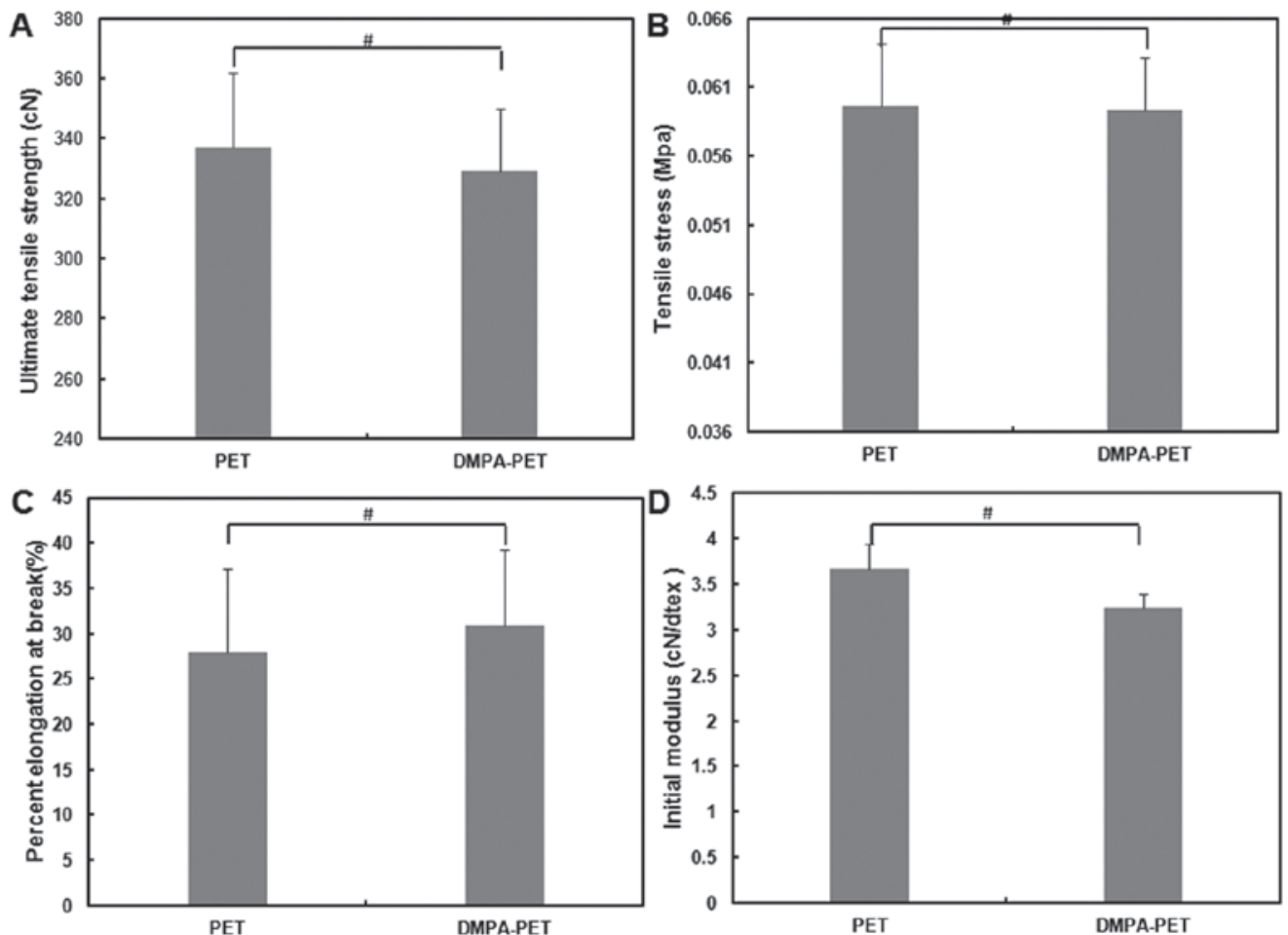

Figure 4. Mechanical testing of the PET and DMPA-PET for (A) ultimate tensile strength, (B) tensile stress, (C) elongation percentage at breakage, (D) initial modulus ( $\mathrm{P}>0.05, \mathrm{n}=10$ ). PET, polyethylene terephthalate; DMPA-PET, dimethylolpropionic acid-polyethylene terephthalate. 

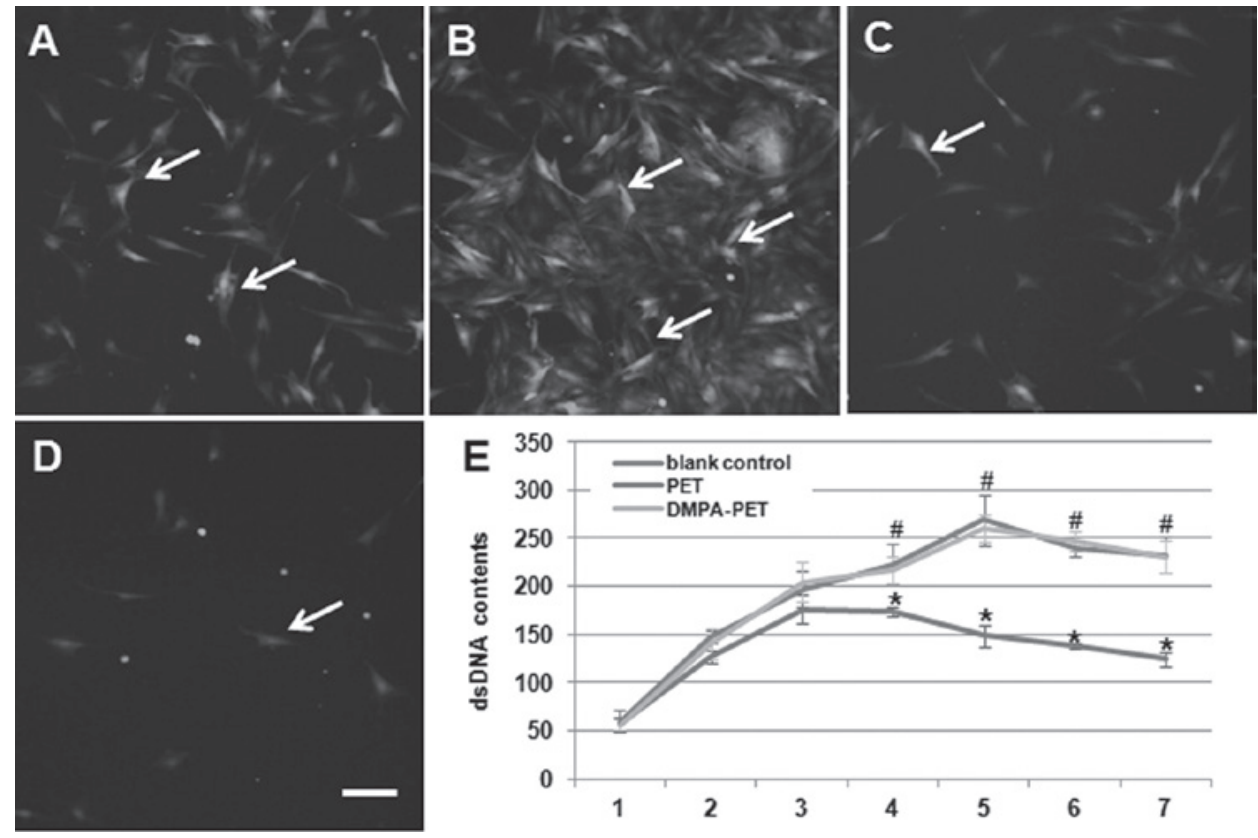

Figure 5. Fluorescence images of the BMSCs on the different fibers: (A) DMPA-PET after 3 days, (B) DMPA-PET after 7 days, (C) PET after 3 days, (D) PET after 7 days (arrows, cells; bar, 50 $\mu \mathrm{m}$ ). (E) DsDNA contents of the different groups $\left({ }^{*} \mathrm{P}<0.05,{ }^{\#} \mathrm{P}>0.05\right.$, $\mathrm{n}=10$ ). BMSCs; bone marrow stromal cells; PET, polyethylene terephthalate; DMPA-PET, dimethylolpropionic acid-polyethylene terephthalate; dsDNA, double-stranded DNA.

(336.8 $\pm 25.15 \mathrm{cN}$ and $0.05966 \pm 0.00445 \mathrm{MPa}$, respectively; $\mathrm{n}=10, \mathrm{P}>0.05$; Fig. 4A and B). Although the elongation percentage at breakage of the samples from the DMPA-PET group $(30.9 \pm 8.4 \%)$ was slightly higher than that from the PET group (27.9 $\pm 9.2 \%)$, there was no significant difference between them $(n=10, P>0.05$; Fig. $4 C)$. The initial modulus of the DMPA-PET group $(3.234 \pm 0.1530 \mathrm{cN} /$ dtex $)$ was a little lower than that of the PET group $(3.661 \pm 0.2734 \mathrm{cN} / \mathrm{dtex})$, but the difference was not significant ( $n=10, P>0.05$; Fig. 4D).

Cytocompatibility evaluation. Using inverted phase-contrast fluorescence microscopy, we found that the BMSCs seeded onto the DMPA-PET fibers appeared flattened, polygonal, spindle-shaped and evenly distributed after 3 days of culture (Fig. 5A). On the 7th day, a number of cells were spread and colonized patches appeared on the surface of DMPA-PET (Fig. 5B). By contrast, the number of cells that had adhered to the surface of the PET fibers on the 3rd day had decreased (Fig. 5C). On the 7th day, only a few cells were found on the surface of PET (Fig. 5D). The dsDNA content of the DMPA-PET group gradually increased from days 1 to 5, with a slight decline from days 5 to 7 after confluence. There were no differences in the dsDNA content between the DMPA-PET group and the blank control group ( $n=10, P>0.05$; Fig. 5E). On the contrary, the dsDNA content of the PET group increased very slowly, and began to decrease from the 4th day. The dsDNA content of the PET group was much lower than that of the DMPA-PET group and the blank control group after day $4(n=10, P<0.05$; Fig. 5E).

\section{Discussion}

PET is commercially one of the most significant and successful engineered polymers, and is widely used in the form of fibers, films and molded articles (27). Due to its high plasticity, flexibility and mechanical resistance to rupturing, PET fibers are considered to be especially adapted to fabricate artificial ligaments (22). The Ligament Advanced Reinforcement System (LARS) ligament, which is made of PET, has become one of the graft choices for ligament rupture management, along with autografts and allografts, in a number of countries (15). However, as PET fibers are hydrophobic, certain clinical studies have proven that LARS ligaments integrate poorly into the surrounding host tissue resulting in failed ligament reconstruction after long-term follow-up (28). Thus, a number of studies have focused on modifying the surface of PET by grafting functional chemical groups, including carboxylic, amide, sulfonate and hydroxyapatite (21,29). However, since PET is semi-crystalline in nature and does not contain chemically reactive groups, it is resistant to straight chemical modification (27).

In order to improve the biocompatibility of PET, we developed a novel modification method involving 3 steps. Firstly, we treated PET fibers with sodium hydroxide to hydrolyze certain esters on the surface of the fibers. Secondly, the hydrolyzed PET fibers were reacted with TDI in acetone solution with DBTDL as the activator. Finally, the fibers were reacted with a hydrophilic molecule, DMPA, with fluorate boron as the activator. The excess base was removed by repeated washing with distilled water until the $\mathrm{pH}$ of the solution returned to a physiological range (7.0-7.4). The SEM images demonstrated that plenty of granules were deposited on the surface of the fibers after DMPA modification (Fig. 1B). This observed difference was attributed to the fragmentation of polymer chains caused by surface etching, and the deposition of grafted components (DMPA) on the filament surface. This is a general behavior which has been observed in other systems (30). This 
rough surface was considered to be increasingly suitable for the adhesion of cells (31). The FTIR data further confirmed that a number of hydrophilic groups, including hydroxyl and carboxyl, were formed on the surface of the DMPA-PET fibers (Fig. 2B). Furthermore, from the co-culture with the BMSCs, we proved that the DMPA-PET fibers demonstrated excellent cytocompatibility compared to the original PET fibers (Fig. 5).

DMPA is a unique, trifunctional molecule, which incorporates a hindered, tertiary carboxylic acid group and 2 reactive primary hydroxyls. As a crystalline monomer, DMPA has been proven to be odorless and considered to provide superior advantages, including easy control, non-toxicity and high hydrophilicity in the formulation and processing of water soluble resins (32-35). It can be used as a modifier for urethane, polyester/alkyd, phenolic, amino and epoxy systems (33). The hindered carboxyl of DMPA makes the introduction of free acid groups easy and convenient (34). In this study, we succeeded in improving the hydrophilicity and cytocompatibility of PET fibers by modifying the surface of DMPA.

In order to investigate the effects of DMPA modification on the changes in the thermophysical and crystal properties of the PET fibers, DSC and XRD were employed in this study. Notably, the DSC data demonstrated that there were no significant changes in the endothermic peaks, fusion enthalpy and crystalline indices between the DMPA-PET and the PET fibers, which indicated that the original thermophysical and crystal properties of PET remained in the DMPA-modified PET fibers (Table I). These results were further confirmed by the XRD patterns (Fig. 3B). We considered that the main reason behind these results was that the introduction of DMPA had no effect on the crystalline structure of the PET fibers. In line with our study, DMPA was found to be easily built into the polymer chain without side reactions during the process, and to not influence the natural thermophysical and crystal properties of the final product (34).

In order to observe the effects of DMPA modification on the mechanical strength of PET, the tensile test was applied in this study. The ultimate tensile strength and tensile stress of one single fiber from the DMPA-PET group were similar to those from the PET group. Although the elongation percentage at breakage of the DMPA-PET group was slightly higher than that of the PET group, there was no significant difference between the 2 groups. Similarly, the difference in the initial modulus between the 2 groups was also not significant (Fig. 4). The PET fiber is regarded as an ideal material for the preparation of artificial ligaments, due to its high strength and stiffness, combined with reduced strain-to-failure (25). However, certain modified methods have been found to cause a decrease in the mechanical properties, including tensile strength, elongation at breakage or the elastic modulus of PET during the processing of the modification (28-30). In this study, we proved that our 3-step DMPA-modification method can significantly increase the hydrophilicity of the PET fibers without changing their mechanical strength.

In this study, we modified PET fibers by using DMPA. The characteristics of the modified fibers were observed and compared with those of the original PET. Based on our results, we found that the introduction of DMPA can significantly increase the hydrophilic group on the surface of the PET fibers, which results in an improvement of the biocompatibility without comprising the mechanical strength. These results demonstrate that DMPA-modified PET fibers have significant potential as a material for the development of artificial ligaments.

\section{Acknowledgements}

This study was supported by the National Science Foundation of China (No. 81071457).

\section{References}

1. Söderman K, Pietilä T, Alfredson H, et al: Anterior cruciate ligament injuries in young females playing soccer at senior levels. Scand J Med Sci Sports 12: 65-68, 2002.

2. Keene G: Arthroscopic reconstruction of the anterior cruciate ligament. A comparison of patellar tendon autograft and four-strand hamstring tendon autograft. Am J Sports Med 28: 438, 2000.

3. Mihelic R, Jurdana H, Jotanovic Z, et al: Long-term results of anterior cruciate ligament reconstruction: a comparison with non-operative treatment with a follow-up of 17-20 years. Int Orthop 35: 1093-1097, 2011.

4. Price JS, Till SH, Bickerstaff DR, et al: Degradation of cartilage type II collagen precedes the onset of osteoarthritis following anterior cruciate ligament rupture. Arthritis Rheum 42: 2390-2398, 1999.

5. Montgomery A: Can many anterior cruciate ligament (ACL) ruptures heal without surgery? N Z Med J 124: 104, 2011.

6 . Vavken $\mathrm{P}$ and Murray MM: The potential for primary repair of the ACL. Sports Med Arthrosc 19: 44-49, 2011.

7. Bryant AL, Creaby MW, Newton RU and Steele JR: Hamstring antagonist torque generated in vivo following ACL rupture and ACL reconstruction. Knee 17: 287-290, 2010.

8. Mauch C, Arnold MP, Wirries A, et al: Anterior cruciate ligament reconstruction using quadriceps tendon autograft for adolescents with open physes- a technical note. Sports Med Arthrosc Rehabil Ther Technol 3: 7, 2011.

9. Bak K, Jørgensen U,Ekstrand J and Scavenius M: Reconstruction of anterior cruciate ligament deficient knees in soccer players with an iliotibial band autograft. A prospective study of 132 reconstructed knees followed for 4 (2-7) years. Scand J Med Sci Sports 11: 16-22, 2001.

10. Burks RT, Crim J, Fink BP, et al. The effects of semitendinosus and gracilis harvest in anterior cruciate ligament reconstruction. Arthroscopy 21: 1177-1185, 2005.

11. Lee S, Seong SC, Jo H, et al: Outcome of anterior cruciate ligament reconstruction using quadriceps tendon autograft. Arthroscopy 20: 795-802, 2004.

12. Miller SL and Gladstone JN: Graft selection in anterior cruciate ligament reconstruction. Orthop Clin North Am 33: 675-683, 2002.

13. Akhtar MA, Bhattacharya R, Ohly N, et al: Revision ACL reconstruction-causes of failure and graft choices. Br J Sports Med 45: A15-A16, 2011.

14. Díaz-de-Rada P, Barriga A, Barroso JL, et al: Positive culture in allograft ACL-reconstruction: what to do? Knee Surg Sports Traumatol Arthrosc 11: 219-222, 2003.

15. Legnani C, Ventura A, Terzaghi C, et al: Anterior cruciate ligament reconstruction with synthetic grafts. A review of literature. Int Orthop 34: 465-471, 2010.

16. Matsumoto $H$ and Fujikawa K: Leeds-Keio artificial ligament: a new concept for the anterior cruciate ligament reconstruction of the knee. Keio J Med 50: 161-166, 2001.

17. Bernardino S: ACL prosthesis: any promise for the future? Knee Surg Sports Traumatol Arthrosc 18: 797-804, 2010.

18. Pavon-Djavid G, Gamble LJ, Ciobanu M, et al: Bioactive poly (ethylene terephthalate) fibers and fabrics: grafting, chemical characterization, and biological assessment. Biomacromolecules 8: 3317-3325, 2007.

19. Bolton CW and Bruchman B: Mechanical and biological properties of the GORE-TEX expanded polytetrafluoroethylene (PTFE) prosthetic ligament. Aktuel Probl Chir Orthop 26: 40-51, 1983. 
20. Lavoie P, Fletcher J and Duval N: Patient satisfaction needs as related to knee stability and objective findings after ACL reconstruction using the LARS artificial ligament. Knee 7: 157-163, 2000.

21. Zhou J, Ciobanu M, Pavon-Djavid G, et al: Morphology and adhesion of human fibroblast cells cultured on bioactive polymer grafted ligament prosthesis. Conf Proc IEEE Eng Med Biol So: 5115-5118, 2007

22. Ventura A, Terzaghi C, Legnani C, et al: Synthetic grafts for anterior cruciate ligament rupture: 19-year outcome study. Knee 17: 108-113, 2010.

23. Carson L, Kelly-Brown C, Stewart M, et al: Synthesis and characterization of chitosan-carbon nanotube composites. Mater Lett 63: 617-620, 2009.

24. Hadjizadeh A, Ajji A and Bureau MN: Nano/micro electro-spun polyethylene terephthalate fibrous mat preparation and characterization. J Mech Behav Biomed Mater 4: 340-351, 2011.

25. Lechat C, Bunsell A, Davies P and Piant A: Mechanical behaviour of polyethylene terephthalate and polyethylene naphthalate fibres under cyclic loading. J Mater Sci 41: 1745-1756, 2006.

26. Peng $\mathrm{CH}$, Bai BS and Chen YF: Study on the preparation of $\mathrm{Mn}-\mathrm{Zn}$ soft magnetic ferrite powders from waste $\mathrm{Zn}-\mathrm{Mn}$ dry batteries. Waste Manag 28: 326-332, 2008

27. Abdolahifard M, Hajir Bahrami S and Malek R: Surface modification of PET fabric by graft copolymerization with acrylic acid and its antibacterial properties. ISRN Organic Chemistry Article ID 265415, 2011
28. Guidoin MF, Marois Y, Bejui J, et al: Analysis of retrieved polymer fiber based replacements for the ACL. Biomaterials 21: 2461-2474, 2000

29. Li H, Ge Y, Wu Y, et al: Hydroxyapatite coating enhances polyethylene terephthalate artificial ligament graft osseointegration in the bone tunnel. Int Orthop 35: 1561-1567, 2011.

30. Gupta B, Revagade N, Atthoff B, et al: Radiation-induced graft modification of knitted poly(ethylene terephthalate) fabric for collagen immobilization. Polym Adv Technol 18: 281-285, 2007.

31. Pezzatini S, Morbidelli L, Gristina R, et al: A nanoscale fluorocarbon coating on PET surfaces improves the adhesion and growth of cultured coronary endothelial cells. Nanotechnology 19: 275101, 2008

32. Zia KM, Zuber M, Barikani M, et al: Surface characteristics of chitin-based shape memory polyurethane elastomers. Colloids Surf B Biointerfaces 72: 248-252, 2009.

33. Narayan R, Chattopadhyay DK, Sreedhar B, et al: Synthesis and characterization of crosslinked polyurethane dispersions based on hydroxylated polyesters. J Appl Polym Sci 99: 368-380, 2006.

34. Rahman MM, Kim EY and Lee WK: Effect of DMPA-clay-POSS content on thermal and mechanical properties of nanostructured ionomeric polyurethanes. J Nanosci Nanotechnol 10: 6981-6985, 2010.

35. Ren Z, Ma D, Wang Y and Zhao G: Molecular structure and hydrogen bonds in solid dimethylol propionic acid (DMPA). Spectrochim Acta A Mol Biomol Spectrosc 59: 2713-2722, 2003. 